\title{
Isothermal Crystallization Kinetics Effect on the Tensile Properties of PLA/PTT Polymer Composites
}

\author{
C.-Y. Tsou, ${ }^{a}$ C.-L. Wu, ${ }^{a}$ Y.-C. Tseng, ${ }^{a}$ S.-H. Chiu, ${ }^{a}$ M.-C. Suen, ${ }^{b}$ W. S. Hung, ${ }^{c}$ and \\ C.-H. Tsou ${ }^{\mathrm{d}, \mathrm{e}, \mathrm{I}}$
}

${ }^{a}$ Department of Materials Science and Engineering, National Taiwan University of Science and Technology, Taipei, Taiwan

${ }^{\mathrm{b}}$ Lee-Ming Institute of Technology, Department of Fashion Business Administration, Taishan, New Taipei City, Taiwan (R.O.C.)

${ }^{\mathrm{c}}$ R\&D Center for Membrane Technology, Chung Yuan University, Chung Li, Taiwan

${ }^{\mathrm{d}}$ Institute of Materials Science and Engineering, Material Corrosion and Protection Key Laboratory of Sichuan Province, Sichuan University of Science and Engineering, Zigong, China

${ }^{\mathrm{e}}$ Faculties of Biological and Chemical Engineering, Faculties of Materials Engineering, Science and Technology Innovation Center, Panzhihua University, Panzhihua, China

1.mayko0301@hotmail.com

A poly(lactic acid)/poly(trimethylene terephthalate) (PLA/PTT) composite was prepared by melt blending to improve the PTT crystallization rate. Morphology analysis of PLA/PTT fractured surfaces demonstrated the compatibility of its components. Thermogravimetric analysis revealed that the thermodegradation of a PLA/PTT sample was higher than that of PLA. Differential scanning calorimetry was used to evaluate the crystallization behavior. The Avrami equation described the isothermal crystallization kinetics. The Hoffman-Weeks parameters indicated that the PLA presence increased slightly the PTT nucleation. The tests of PLA, PTT, and PLA/PTT specimens in tension showed that a percent elongation of the PLA/PTT composite was between that of PLA and PTT; however, the tensile strength of the PLA/PTT composite was similar to that of PLA.

Keywords: poly(lactic acid), poly(trimethylene terephthalate), tensile properties, nucleation, crystallization.

Introduction. Poly(lactic acid) (PLA) is a biodegradable polymer, differing from most of thermoplastic polymers since it is compounded from such renewable materials, as sugar cane or corn starch. PLA possesses quite promising properties; however, the brittleness of the material restricts its applicability and processing abilities [1]. Poly(trimethylene terephthalate) (PTT) has a structure similar to that of polybutylene terephthalate and polyethylene terephthalate [2]. As compared to these two other terephthalates, PTT demonstrates higher bending strength, which makes it suitable for manufacturing different materials, such as textiles or engineering plastics [2-4]. The brittleness of PLA can be improved by blending it with an elastomer [5-22]. The high thermostability of PTT, blended with PLA [23] provided the conditions for studying the nonisothermal crystallization of PTT/PLA. However, the isothermal crystallization behavior of PLA/PTT composites has not been reported. In the present study, PTT was used to enhance the PLA elongation at break through the melt-compounding method. The Hoffman-Weeks equation was applied to predict the equilibrium melting point $\left(T_{m}^{0}\right)$. The effect of PLA on the melting, crystallization, and thermostability of PLA/PTT specimens was examined. Differential scanning calorimetry (DSC) and wide-angle X-ray diffraction (WXRD) were used to analyze the crystallization behavior of a PLA/PTT composite.

1. Experimental. PLA with a melt flow index of 4-8 g/10 min (Nature Works 2002D) and PTT with a melt flow index of $25 \mathrm{~g} / 10 \mathrm{~min}$ (ASTM-D1238), were supplied by Shell 
Chemical Co. Ltd., USA. PLA/PTT composites (70/30 weight ratio) were prepared using a Brabender at $50 \mathrm{rpm}$ and $250^{\circ} \mathrm{C}$ for $4 \mathrm{~min}$. PLA, PTT, and PLA/PTT composites were studied by thermogravimetric analysis (TGA), DSC, WXRD, and scanning electron microscopy (SEM). TGA was run in the range of 30 to $650^{\circ} \mathrm{C}$ at a heating rate of $20^{\circ} \mathrm{C} / \mathrm{min}$. In DSC, the first round was at a rate of $100^{\circ} \mathrm{C} / \mathrm{min}$ within $0-250^{\circ} \mathrm{C}$ for $3 \mathrm{~min}$ to avoid thermal history effects, followed by a decrease from $250^{\circ} \mathrm{C}$ to $20^{\circ} \mathrm{C}$ at a rate of $5,10,20$, and $30^{\circ} \mathrm{C} / \mathrm{min}$, respectively, to observe the melt crystallization. WXRD spectra were obtained using a Rigaku model RU-H3R. The data were recorded over the range of 10 to $35^{\circ}$ with a scanning interval of $0.05^{\circ}$.

\section{Results and Discussion.}

2.1. Thermogravimetric Analysis. The TGA curves for PLA, PTT, and PLA/PTT are shown in Fig. 1. The PLA thermostability was improved by the PTT addition, having the higher thermostability. Table 1 lists TGA values, demonstrating that at 5 and $10 \mathrm{wt} \%$ losses, the temperature of the composite was considerably higher than that of each constituent due to the PTT addition to the blend.

$\mathrm{T}$ a b 1 e 1

TGA of PLA, PTT, and PLA/PTT Samples

\begin{tabular}{|c|c|c|c|}
\hline Specimen & $T_{g}$ at $5 \mathrm{wt} \%\left({ }^{\circ} \mathrm{C}\right)$ & $T_{g}$ at $10 \mathrm{wt} . \%\left({ }^{\circ} \mathrm{C}\right)$ & Char residue at $600{ }^{\circ} \mathrm{C}(\%)$ \\
\hline PLA & 282.4 & 311.6 & 0.57 \\
PTT & 369.9 & 379.7 & 13.35 \\
PLA/PTT & 326.7 & 335.9 & 11.33 \\
\hline
\end{tabular}

Note. Heating rate $=20^{\circ} \mathrm{C} / \mathrm{min}$.

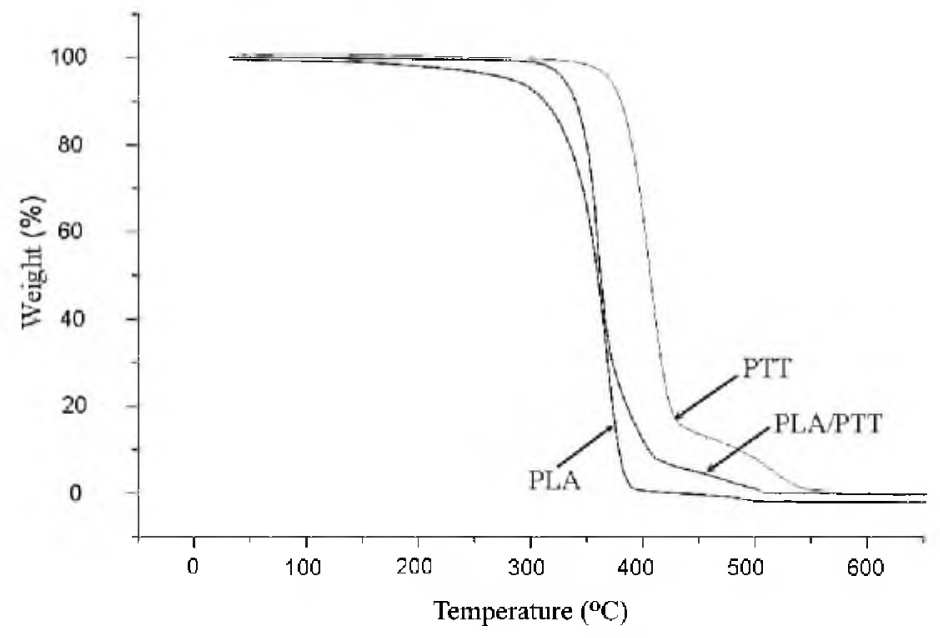

Fig. 1. TGA curves for PLA, PTT, and PLA/PTT.

2.2. Differential Scanning Calorimetry. The DSC data for a heating rate of $20^{\circ} \mathrm{C} / \mathrm{min}$ are presented in Fig. 2. The $T_{g}$ of PLA in the composite became a little higher with the addition of PTT. A slight cold crystallization of the PLA/PTT composite might apparently be due to the partial PTT and PLA compatibility. The nonisothermal crystallization of PLA, PTT, and PLA/PTT was studied with DSC. The cooling rate ranged from 5 to $30^{\circ} \mathrm{C} / \mathrm{min}$. The crystallinity data for PLA, PTT, and PLA/PTT samples at different cooling rates are summarized in Table 2. As is seen, PLA exhibited no crystallinity because of its slow crystallization rate, neat PLA was unable to crystallize. To examine the variation of the 
$\mathrm{T}$ a b 1 e 2

DSC Values for PLA, PTT, and PLA/PTT at Different Cooling Rates

\begin{tabular}{||c|c|c|c|c||}
\hline Sample & $\begin{array}{c}\text { Cooling rate } \\
\left({ }^{\circ} \mathrm{C} / \mathrm{min}\right)\end{array}$ & $T_{m c},{ }^{\circ} \mathrm{C}$ & $\Delta H_{m}, \mathrm{~J} / \mathrm{g}$ & $X_{t}, \%$ \\
\hline \multirow{2}{*}{ PTT } & 5 & 196.7 & 45.4 & 45.4 \\
& 10 & 191.8 & 45.0 & 45.0 \\
& 20 & 190.6 & 43.2 & 43.2 \\
& 30 & 180.5 & 40.8 & 40.8 \\
\hline \multirow{2}{*}{ PLA/PTT } & 5 & 198.9 & 19.6 & 65.3 \\
& 10 & 193.1 & 15.3 & 51.0 \\
& 20 & 191.8 & 14.4 & 47.7 \\
& 30 & 186.3 & 12.3 & 41.0 \\
\hline
\end{tabular}

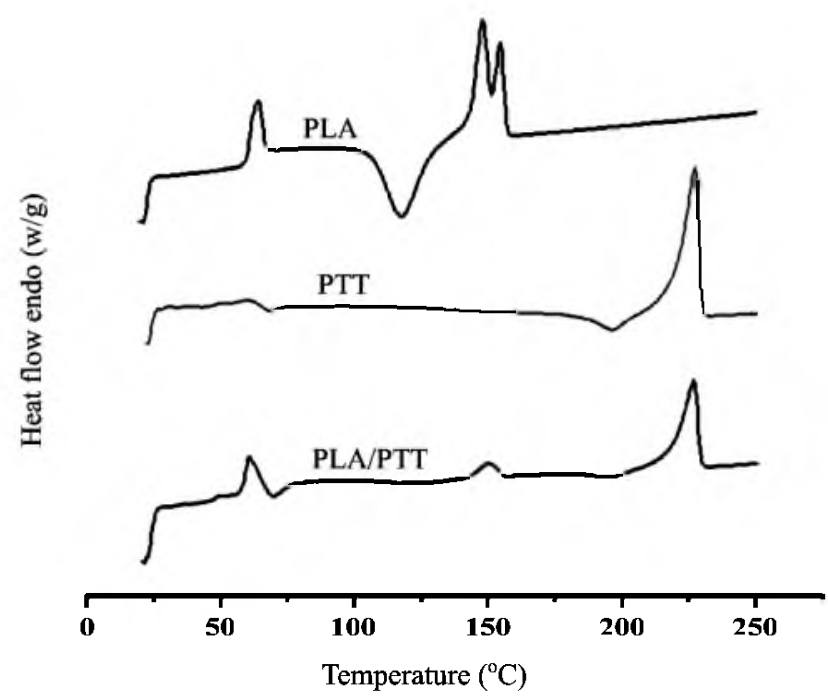

Fig. 2. DSC for PLA, PTT, and PLA/PTT (heating rate $=20^{\circ} \mathrm{C} / \mathrm{min}$ ).

crystallization degree of PTT and PLA/PTT samples, depending on the cooling rate $\left(5-30^{\circ} \mathrm{C} / \mathrm{min}\right)$, the crystallinity $\left(X_{t}\right)$ of all the samples was determined from the peak areas. The enthalpy is determined as

$$
X_{t}=\left(\Delta H_{(D S C)} / \Delta H_{(100 \%)}\right)(100 / W)
$$

where $\Delta H_{(100 \%)}$ PTT $=145.48 \mathrm{~J} / \mathrm{g}$ [24] and $W$ is the weight fraction of PTT in the sample. For PTT and a PLA/PTT composite, $X_{t}$ decreased with a cooling rate. The data for the nonisothermal crystallization are listed in Table 3. The melt crystallization temperature $\left(T_{m c}\right)$ and $X_{t}$ of the PLA/PTT composite is higher than that of neat PTT. The PLA component in the PLA/PTT composite can enhance the PTT crystallization rate.

The relative crystallinity of the PLA/PTT composite at several isothermal crystallization temperatures is given in Fig. 3. The crystallization requires mere nucleation and accumulation of the molecular chains, although higher isothermal crystallization temperatures hinder nucleation because the molecular chain motion is easier. Therefore, the nucleation can be controlled; at low temperatures, the nucleation can easily be reached, but the molecular motion is more difficult. 
T a b 1 e 3

Avrami Parameters for Various Crystallization Temperatures

\begin{tabular}{|c|c|c|c|c|c|c|c|}
\hline Sample & $T_{c},{ }^{\circ} \mathrm{C}$ & $\Delta H, \mathrm{~J} / \mathrm{g}$ & $X_{t}, \%$ & $t_{1 / 2}, \min$ & $n$ & $K, \mathrm{~s}^{-1}$ & $R^{2}$ \\
\hline \multirow{2}{*}{ PTT } & 197 & 50.5 & 50.6 & 0.46 & 2.21 & 3.894 & 0.998 \\
& 199 & 48.6 & 48.7 & 0.71 & 2.27 & 1.801 & 0.997 \\
& 201 & 45.3 & 45.5 & 1.04 & 2.55 & 0.676 & 0.998 \\
& 203 & 41.4 & 41.4 & 1.51 & 2.73 & 0.173 & 0.999 \\
& 205 & 40.5 & 40.5 & 2.06 & 2.85 & 0.041 & 0.997 \\
\hline \multirow{2}{*}{ PLA/PTT } & 197 & 17.2 & 57.3 & 0.71 & 2.17 & 1.508 & 0.999 \\
& 199 & 16.0 & 53.3 & 0.86 & 2.27 & 0.916 & 0.998 \\
& 201 & 14.1 & 47.0 & 1.21 & 2.45 & 0.391 & 0.999 \\
& 203 & 13.1 & 43.7 & 1.81 & 2.45 & 0.134 & 0.997 \\
& 205 & 12.3 & 41.0 & 2.67 & 2.50 & 0.045 & 0.999 \\
\hline
\end{tabular}

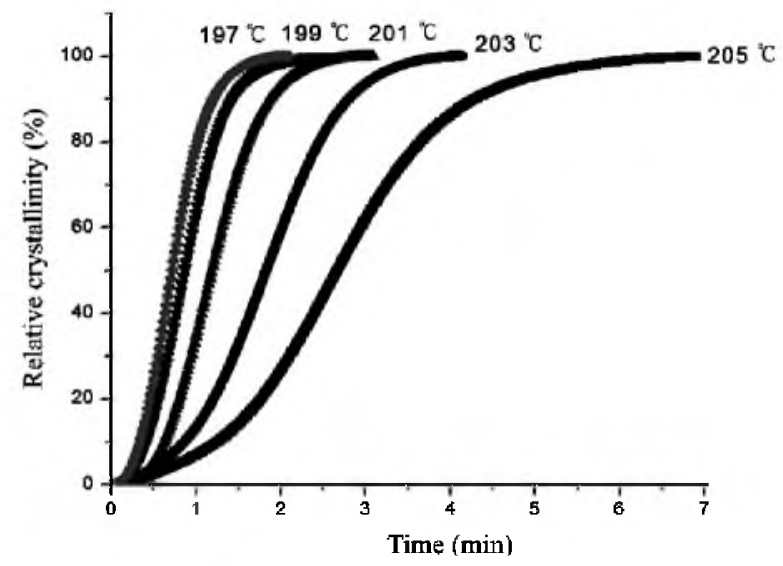

Fig. 3. Relative crystallinity of PLA/PTT.

The Avrami calculation defined the isothermal crystallization kinetics of PTT, PTT, and PLA/PTT samples [25]. The relation between $X_{t}$ and time $t$ is indicated by

$$
1-X_{t}=\exp \left(-k t^{n}\right)
$$

Taking the double natural logarithm of this calculation, we get

$$
\ln \left[-\ln \left(1-X_{t}\right)\right]=n \ln t+\ln k,
$$

where $n$ is the Avrami exponent and $k$ is the kinetic parameter of the isothermal crystallization. Equation (3) was used to plot the linear curves for PLA/PTT data in Fig. 4; the slope was equal to the Avrami exponent $n$ for the $y$-intercept corresponding to the kinetic parameter $(k)$. Those values for all samples are cited in Table 3.

The $X_{t}$ value of the PLA/PTT composite shows unessential differences at varying crystallization temperatures. The Avrami exponents $n$ for the PLA/PTT composite were within 2.0-3.0. Dissimilar $n$ values indicate unlike crystalline forms and nucleation of crystals. The $t_{1 / 2}$ value for isothermal crystallization was deduced from the equation:

$$
t_{1 / 2}=(\ln 2 / k)^{1 / n}
$$




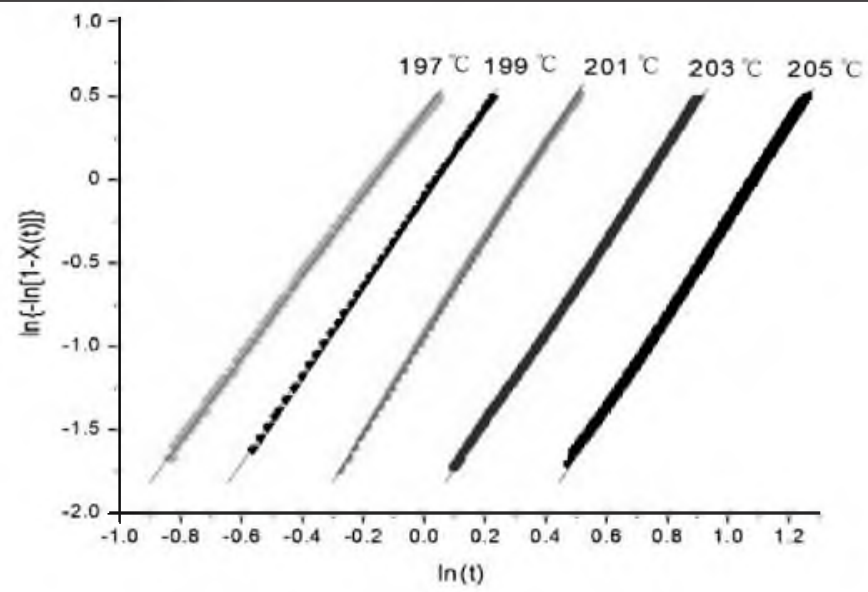

Fig. 4. Avrami plots for PLA/PTT samples at different isothermal crystallization temperatures.

The $k$ value for all the samples declined with the isothermal crystallization temperature, which is due to the lower viscosity at higher temperatures; thus, the kinetic energy of the polymer was growing. However, increasing entropy inhibits the formation of crystal nuclei, causing a decrease in the crystal growth. In contrast, the $t_{1 / 2}$ values for all the samples increased with temperature. The presence of PLA hinders the motion of PTT molecular chains; then, the $k$ value for PTT was reduced due to PLA.

The Hoffman-Weeks equation

$$
T_{m}=\left(T_{c} / \gamma\right)+(1-1 / \gamma) T_{m}^{0}
$$

is usually applied to estimate the $T_{m}^{0}$ value of semicrystalline materials. The melting point $\left(T_{m}\right)$ of the main crystallization process was set as $T_{m}^{0}$ because of numerous melting peaks appearing during the PTT melting [26]. The $T_{m}^{0}$ value can be obtained from the relationship for $T_{c}$ and $T_{m}$ (Fig. 5). In the scheme, linear regression analysis for numerous points was performed, and by extrapolation, the regression line intersected with the line $T_{m}=T_{c}$.

As is shown in Table 4, $T_{m}^{0}$ for the PLA/PTT composite was slightly higher than $T_{m}^{0}$ of neat PTT. The morphology factor $\gamma=L / L^{*}$, which is the thickness ratio of molecular chains. With known $T_{m}^{0}$, the difference in the crystallization regime can be found from the Lauritzen-Hoffman equation for the spherulite growing rate:

$$
\begin{gathered}
G=G_{0} \exp \left[-U^{*} / R\left(T_{c}-T_{\infty}\right)\right] \exp \left[-K_{g} / T_{c}(\Delta T) f\right], \\
\left(1 / t_{1 / 2}\right)=\left(1 / t_{1 / 2}\right)_{0} \exp \left[-U^{*} / R\left(T_{c}-T_{\infty}\right)\right] \exp \left[-K_{g} / T_{c}(\Delta T) f\right] .
\end{gathered}
$$

Tsou [18] substituted $\left(1 / t_{1 / 2}\right)$ and $\left(1 / t_{1 / 2}\right)_{0}$ for $G$ and $G_{0}$, respectively, and obtained

$$
K_{g}=n \sigma \sigma_{e} T_{m}^{0} / \Delta h_{j} k_{B}
$$

The linear regression results on the logarithmic scale are demonstrated in Fig. 6, $U^{*}=4.12 \mathrm{kcal} / \mathrm{mole}, T_{\infty}=T_{g}-51.6(\mathrm{~K}), f=2 T_{c} /\left(T_{m}^{0}+T_{c}\right), f$ is the correction factor. 
$\mathrm{T}$ a b 1 e 4

Hoffman-Weeks and Lauritzen-Hoffman Parameters for PTT and PLA/PTT

\begin{tabular}{|c|c|c|c|}
\hline Sample & $T_{m}^{0},{ }^{\circ} \mathrm{C}$ & $\gamma$ & $R^{2}$ \\
\hline PLA & - & - & - \\
\hline PTT & 236.2 & 1.92 & 0.988 \\
\hline PLA/PTT & 240.1 & 1.75 & 0.996 \\
\hline
\end{tabular}

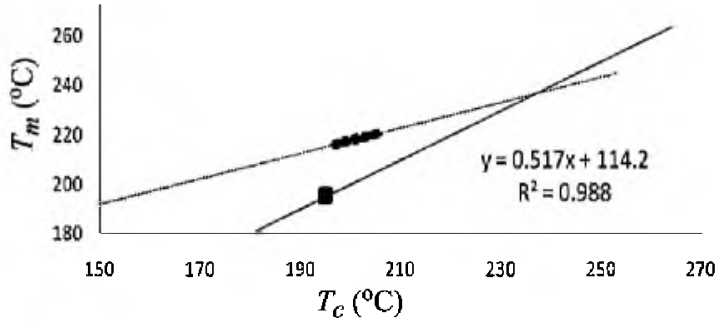

a

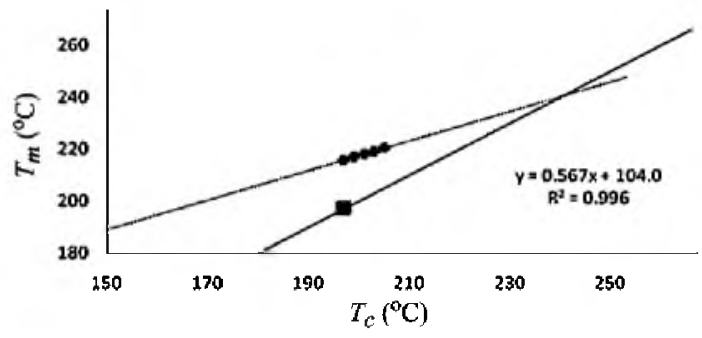

b

Fig. 5. Hoffman-Weeks plots for PTT (a) and PLA/PTT (b).

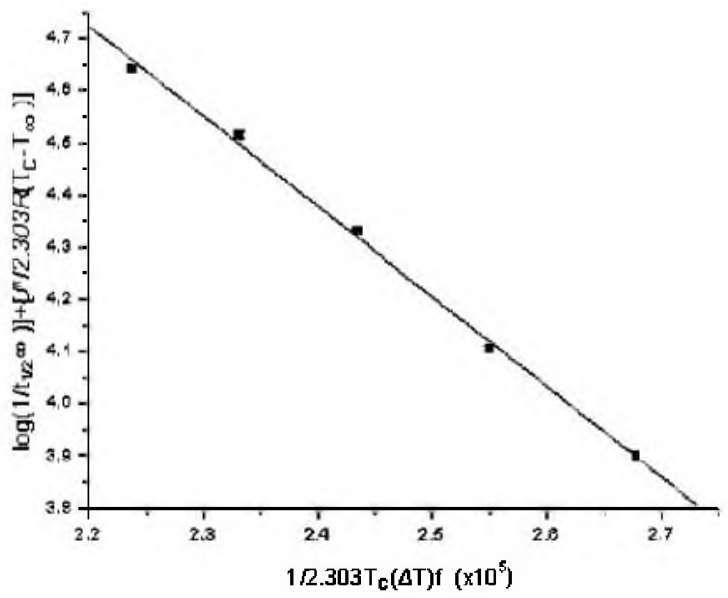

Fig. 6. Lauritzen-Hoffman plots for PLA/PTT.

The slope variations reflected the difference in the crystallization regime. Regime II proceeded at $190-210^{\circ} \mathrm{C}[18]$; the slope $-K_{g} \cdot 10^{5}$ was about -1.68 . The nucleation mechanism for both PTT and the PLA/PTT composite was according to regime II. 
$\mathrm{T}$ a b 1 e 5

Hoffman-Weeks and Lauritzen-Hoffman Parameters for PTT and PLA/PTT

\begin{tabular}{|c|c|c|c|c|}
\hline Samples & $K_{g}$ & Regime & $\sigma_{e}$ & $R^{2}$ \\
PLA & - & - & - & - \\
PTT & 1.480 & II & 58.5 & 0.998 \\
\hline PLA/PTT & 1.731 & II & 61.4 & 0.989 \\
\hline
\end{tabular}

Equation (7) was used to generate the data for $K_{g}$ listed in Table 5, $K_{g}$ values, representing the slopes, were found from the regression lines. The higher $K_{g}$ value corresponds to the better nucleation capability $[27,28]$. Consequently, PLA at $70 \%$ loading slightly enhanced the PTT nucleation.

2.3. WXRD. The X-ray diffraction peak positions for PLA, PTT, and PLA/PTT are shown in Fig. 7. The peaks for PLA were obscrved at the angles $2 \theta$ of approximately 16.6, 19 , and $22.2^{\circ}$, which correspond to the reflection planes of $(1-1-0),(2-0-3)$, and $(1-0-5)$, respectively [28]. The PTT peaks were detected at the angles $2 \theta$ of approximately $27.4,24.7$, and $23.5^{\circ}$, which correspond to the reflection planes of $(1-0-4),(1-1-3)$, and $(1-0-2)$, respectively [29]; and at other angles $2 \theta$ of approximately $21.7,19.5,16.7$, and $15.2^{\circ}$, which are consistent with the reflection planes of $(-1-0-2),(0-1-2),(0-1-2)$, and $(0-1-0)$, respectively [29]. However, the PLA/PTT composite exhibited the characteristic peaks of both PLA and PTT, implying that PLA does not change the PTT crystal forms.

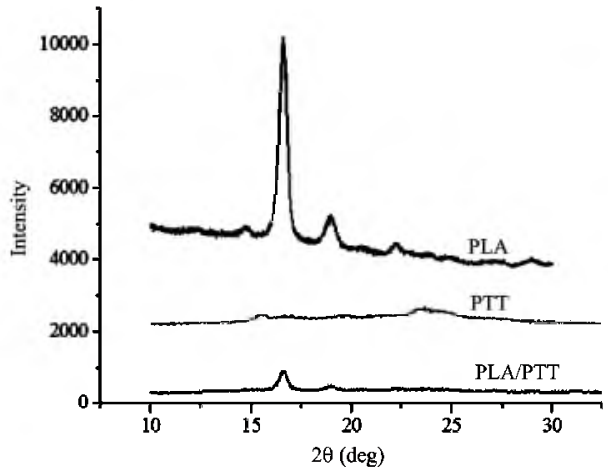

a

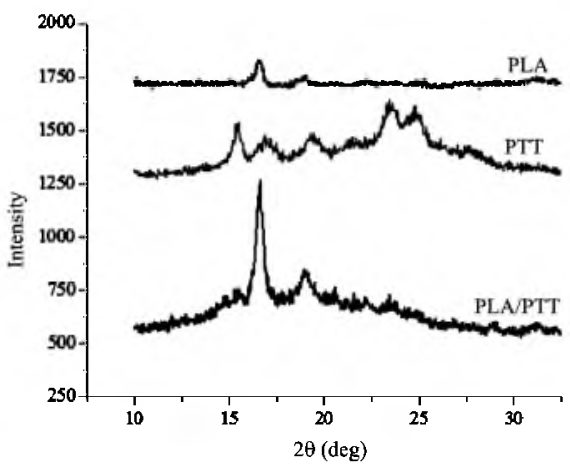

$\mathrm{b}$

Fig. 7. Wide-angle X-ray diffraction for PTT, PLA, and PLA/PTT: (a) cold crystallization and (b) melt crystallization.

2.4. Morphology. The SEM images of the fractured surfaces of PLA, PTT, and PLA/PTT are illustrated in Fig. 8. The fractured surface of PLA had a smooth morphology (Fig. 8a). After blending PLA with PTT, some PTT pellets dispersed in the PLA matrix (Fig. 8c). However, the pellets were not secn clearly because the PTT molecules are partially compatible with the PLA oncs.

2.5. Tensile Properties. The tensile properties of PLA, PTT, and PLA/PTT are depicted in Fig. 9. The elongation at break for PLA was only 4.1\%. After blending PLA with PTT, the PLA/PTT composite revealed a substantial increase in the elongation at break $(6.2 \%)$ since PTT is rather clastic and imparts toughness to PLA. Furthermore, the tensile strength of PLA was similar to that of the PLA/PTT compositc; this might be because of the partial compatibility of PLA and PTT. 


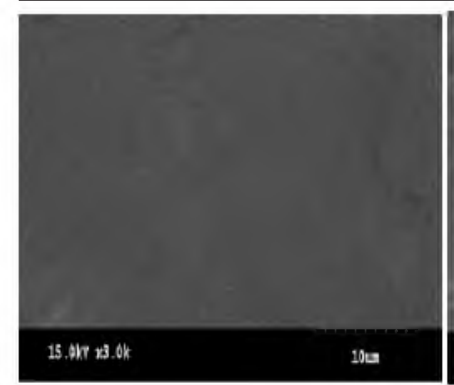

a

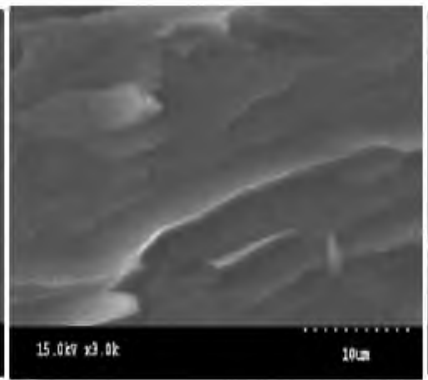

b

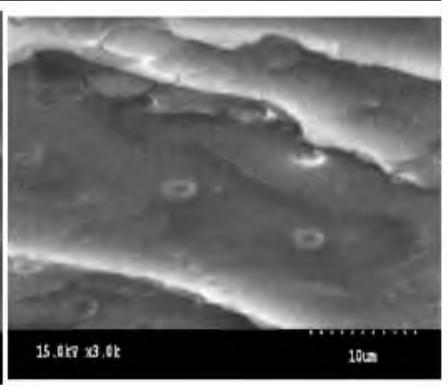

c

Fig. 8. SEM pictures of PLA (a), PTT (b), and PLA/PTT (c).

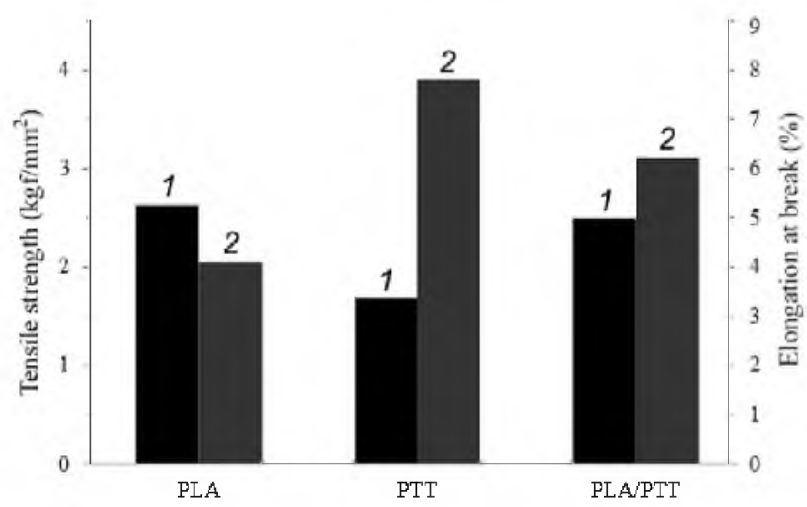

Fig. 9. Tensile strength (I) and elongation at break (2) of PLA, PTT, and PLA/PTT.

Conclusions. The TGA curves revealed that the thermostability of PLA/PTT composites was higher than that of pure PLA because of the higher thermostability of PTT. In addition, the presence of PLA in the PLA/PTT composites slightly increased the PTT nucleation. Therefore, PLA can increase the crystallization rate of PTT in the PLA/PTT composites. WXRD indicated that PLA did not change the typical peaks and crystal forms of PTT. Furthermore, the elongation at break of PLA was enhanced with the PTT addition, and the tensile strength of the PLA/PTT composite was similar to that of PLA because of the partial compatibility of PLA and PTT.

1. S.-L. Yang, Z.-H. Wu, W. Yang, et al., "Thermal and mechanical properties of chemical cross-linked polylactide (PLA)," Polym. Test., 27, No. 8, 957-963 (2008).

2. X. He and D. Yang, "Banded spherulites grown from poly(trimethylene terephthalate) solution-cast film," J. Wuhan Univ. Technol., 23, No. 6, 791-794 (2008).

3. H. Chuah, "Orientation and structure development in poly(trimethylene terephthalate) tensile drawing," Macromolecules, 34, No. 20, 6985-6993 (2001).

4. S. Grebowicz, H. Brown, H. Chuah, et al., "Deformation of undrawn poly(trimethylene terephthalate) (PTT) fibers," Polymer, 42, No. 16, 7153-7160 (2001).

5. J. Zhang, "Study of poly(trimethylene terephthalate) as an engineering thermoplastics material," J. Appl. Polym. Sci., 91, No. 3, 1657-1666 (2004).

6. C.-H. Tsou, W.-S. Hung, C.-S. Wu, et al., "New composition of maleic-anhydridegrafted poly(lactic acid)/rice husk with methylenediphenyldiisocyanate," Mater. Sci.Medzg., 20, No. 4, 446-451 (2015). 
7. H. B. Ravikumar, C. Ranganathaiah, G. N. Kumaraswamy, and S. Thomas, "Positron annihilation and differential scanning calorimetric study of poly(trimethylene terephthalate)/EPDM blends," Polymer, 46, No. 7, 2372-2380 (2005).

8. C.-H. Tsou, H.-T. Lee, M. De Guzman, et a1., "Synthesis of biodegradable polycaprolactone/polyurethane by curing with $\mathrm{H}_{2} \mathrm{O}$," Polym. Bull., 72, No. 7, 15451561 (2015).

9. A. J. Oshinski, H. Keskkula, and D. R. Paul, "Rubber toughening of polyamides with functionalized block copolymers: 1. Nylon-6," Polymer, 33, No. 2, 268-283 (1992).

10. C.-H. Tsou, H.-T. Lee, W.-S. Hung, et al., "Synthesis and properties of antibacterial polyurethane with novel Bis(3-pyridinemethanol) silver chain extender," Polymer, 85, 96-105 (2016).

11. C.-H. Tsou, H.-T. Lee, H.-A. Tsai, et al., "Synthesis and properties of biodegradable polycaprolactone/polyurethanes by using 2,6-pyridinedimethanol as a chain extender," J. Polym. Degrad. Stabil., 98, No. 2, 643-650 (2013).

12. C.-Y. Tsou, C.-L. Wu, C.-H. Tsou, et al., "Biodegradable composition of poly(lactic acid) from renewable wood flour," Polym. Sci. Ser. B+, 57, No. 5, 473-480 (2015).

13. P. A. Tzika, M. C. Boyce, and D. M. Parks, "Micromechanics of deformation in particle-toughened polyamides," J. Mech. Phys. Solids, 48, No. 9, 1893-1929 (2000).

14. C.-H.Tsou, H.-T. Lee, W.-S. Hung, et al., "Effects of different metals on the synthesis and properties of waterborne polyurethane composites containing pyridyl units," Polym. Bull., DOI 10.1007/s00289-016-1767-3 (2016).

15. M.-C. Yang, B.-J. Kao, M.-C. Suen, et al., "The properties and a new preparation of ethylene propylene dienemonomer/montmorillonite nanocomposites," Polym. Polym. Compos., 23, No. 3, 181-190 (2015).

16. C. Zhou, M. Chen, Z. Y. Tan, et al., "The influence of arrangement of St in MBS on the properties of PVC/MBS blends," Eur. Polym. J., 42, No. 8, 1811-1818 (2006).

17. C.-H. Tsou, M.-C. Suen, W.-Y. Wu, et al., "Crystallization behavior and tensile property of poly(trimethyleneterephthalate)/styrene-ethylene-buthylene-styrene composites," J. Wuhan Univ. Technol., 31, No. 2, 474-480 (2016).

18. C.-H. Tsou, M.-C. Suen, W.-H. Yao, et al., "Preparation and characterization of bioplastic-based green renewable composites from tapioca with acetyl tributyl citrate as plasticizer," Materials, 7, No. 8, 5617-5632 (2014).

19. K. Wang, Y. Chen, and Y. Zhang, "Effects of organoclay platelets on morphology and mechanical properties in PTT/EPDM-g-MA/organoclay ternary nanocomposites." Polymer, 49, No. 15, 3301-3309 (2008).

20. D. Juárez, S. Ferrand, O. Fenollar, et al., "Improvement of thermal inertia of styrene-ethylene/butylene-styrene (SEBS) polymers by addition of microencapsulated phase change materials (PCMs)," Eur. Polym. J., 47, No. 2, 153-161 (2011).

21. C.-H. Tsou, B.-J. Kao, M.-C. Yang, et al. "Biocompatibility and characterization of polylactic acid/styrene-ethylene-butylene-styrene composites," Biomed. Mater. Eng., 26, Suppl. 1, S147-S154 (2015).

22. N. R. Savadekar and S. T. Mhaske, "The effect of vulcanized thermoplastics and SEBS on the impact strength of PPT," Polym.-Plast. Technol., 49, No. 15, 1499-1505 (2010).

23. H. Zou, L. Wang, C. Yi, and H. Gan, "Thermal properties and non-isothermal crystallization behavior of poly(trimethylene terephthalate)/poly(lactic acid) blends," Polym. Int., 60, No. 9, 1349-1354 (2011). 
24. M. Pyda, A. Boller, J. Grebowicz, et al., "Heat capacity of poly(trimethylene terephthalate)," J. Polym. Sci. Polym. Phys., 36, No. 14, 2499-2511 (1998).

25. M. Avrami, "Kinetics of phase change. I: General theory," J. Chem. Phys., 7, No. 12 , 1103-1112 (2004).

26. P. Srimoaon, N. Dangseeyun, and P. Supaphol, "Multiple melting behavior in isothermally crystallized poly(trimethylene terephthalate)," Eur. Polym. J., 40, No. 3, 599-608 (2004).

27. S. I. Han, S. W. Kang, B. S. Kim, et al., "A novel polymeric ionomer as a potential biomaterial: Crystallization behavior, degradation, and in-vitro cellular interactions," Adv. Funct. Mater., 15, 367-374 (2005).

28. C.-H. Tsou, B.-J. Kao, M.-C. Suen, et al., "Crystallization behavior and biocompatibility of poly(butylene succinate)/poly(lactic acid) composites," Mater. Res. Innov., 18, No. S2, S2-372-S2-376 (2014).

29. N. Dangseeyun, P. Supaphol, and M. Nithitanakul, "Thermal, crystallization, and rheological characteristics of poly(trimethylene terephthalate)/poly(butylene terephthalate) blends," Polym. Test., 23, No. 2, 187-194 (2004).

Received 30. 08. 2016 